\section{Recent patents in microbiomes and microbial genomics}

Patent number

US 9,610,307

Pharmaceutical compositions containing microbial entities; may be used in conjunction with one or more prebiotics to treat or prevent disorders of the local or systemic microbiome in a subject.

US 9,585,920 Administration of beneficial bacteria to an individual's microbiome that have been modified so as to produce effective amounts of desired compositions, compounds, agents, e.g., tomatidine, p53 protein, etc., is employed to address cancerous conditions. The administration of such beneficial bacteria and microbes to an individual's microbiome invokes either an active (or a passive) immune response to destroy, weaken or render less invasive certain cancerous cells, and preferably maintains muscle tissue to combat cancer cachexia.

US 9,486,487 Methods, systems, compositions, and kits to address the need for microbiome-related treatment of health conditions and disease.

US 9,457,077 A method and system for targeting the microbiome to promote health, involving exposing an expectant mother to immunologic agents and allergens in a fashion (e.g., via exposure to farm animal manure-containing soils) that charges their immune system and that of their fetus(es) so that their babies, once born, are provided with immunity against a variety of autoimmune diseases, including allergies commonly and increasingly experienced in modern urban environments.

US 9,192,361 A fecal microbiome transplant material preparation method and apparatus for the preparation of fecal material for a fecal transplant.

US 9,040,101 Compositions and methods for treating diabetes; in particular, compositions and methods for treating diabetes utilizing a gastrointestinal microbiome-modulating composition.

US 8,980,553 Compositions and methods for enriching non-target polynucleotides from a mixture of non-target and target polynucleotides where differences between the target polynucleotides and the non-target polynucleotides include the extent of modified bases that are present in a greater density in the target polynucleotides than in the non-target polynucleotides. This permits the target polynucleotides to be selectively and rapidly bound to an affinity matrix such as affinity protein-coated magnetic beads providing enrichment of the non-target polynucleotides in the supernatant. One use of this enrichment is to remove human genomic DNA from a mixture of DNAs obtained from human tissue samples to enrich for polynucleotides in a microbiome so as to characterize the microbiome by DNA sequencing.

US 8,713,025 A system, method and computer program product for developing an entity context frame or situation summary before using said context frame/situation summary to develop an index, perform a context search and return prioritized results. The search results may comprise a plurality of health-related data where said health-related data comprise a plurality of microbiome data.

Assignee
Evelo Biosciences
(Cambridge, MA, USA)

Inventor

Date

(Cambridge, MA, USA)

Berry DW, Kaplan J,

4/4/2017

Rahman S

Kovarik KR, Kovarik JE Kovarik KR, Kovarik JE

$3 / 7 / 2017$

Whole Biome (San

Francisco)

Cutcliffe C, Eid JS,

$11 / 8 / 2016$

Bullard JH,

Schicklberger MF

Kovarik KR, Kovarik JE Kovarik KR, Kovarik JE

$10 / 4 / 2016$

Stevens CJ

Stevens CJ

$11 / 24 / 2015$

MicroBiome

Therapeutics

(Broomfield, CO, USA)

New England BioLabs

(Ipswich, MA, USA)

Feehery GR, Stewart F, McFarland J, Pradhan S
Heiman ML, Stull DP, Peno JW

$5 / 26 / 2015$ 